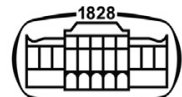

AKADÉMIAI KIADÓ

Acta Veterinaria

Hungarica

$68(2020) 3,263-268$

DOI:

$10.1556 / 004.2020 .00038$

(c) 2020 The Author(s)

RESEARCH ARTICLE

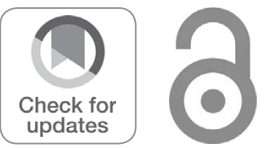

Corresponding author.

E-mail: vincze.boglarka@univet.hu

\section{Family aggregation analysis shows a possible heritable background of equine grass sickness (dysautonomia) in a Hungarian stud population}

\author{
BOGLÁRKA VINCZE ${ }^{1^{*}} \oplus$, MÁRTA VARGA ${ }^{2}$, \\ ORSOLYA KUTASI ${ }^{2}$, PETRA ZENKE ${ }^{2}$, OTTÓ SZENCI ${ }^{1}$, \\ FERENC BASKA ${ }^{3}$, ALAN BARTELS ${ }^{4}$, SÁNDOR SPISÁK ${ }^{4}$, \\ SÁNDOR CSEH ${ }^{1}$ and NORBERT SOLYMOSI ${ }^{5}$
}

\footnotetext{
${ }^{1}$ Department of Obstetrics and Food Animal Medicine Clinic, University of Veterinary Medicine, István u. 2, H-1078 Budapest, Hungary

2 Department of Animal Breeding, Nutrition and Laboratory Animal Science, Laboratory of Genetics, University of Veterinary Medicine, Budapest, Hungary

${ }^{3}$ Department and Clinic of Zoo and Wildlife Medicine, University of Veterinary Medicine, Budapest, Hungary

${ }^{4}$ Department of Medical Oncology, Dana-Farber Cancer Institute, Harvard University, Boston, MA, USA

${ }^{5}$ Centre for Bioinformatics, University of Veterinary Medicine, Budapest, Hungary
}

Received: April 6, 2020 • Accepted: May 8, 2020

Published online: October 30, 2020

\begin{abstract}
Equine grass sickness (also known as dysautonomia) is a life-threatening polyneuropathic disease affecting horses with approx. $80 \%$ mortality. Since its first description over a century ago, several factors, such as the phenotype, intestinal microbiome, environment, management and climate, have been supposed to be associated with the increased risk of dysautonomia. In this retrospective study, we examined the possible involvement of genetic factors. Medical and pedigree datasets regarding 1,233 horses with 49 affected animals born during a 23-year period were used in the analysis. Among the descendants of some stallions, the proportion of animals diagnosed with dysautonomia was unexpectedly high. Among males, the odds of dysautonomia were found to be higher, albeit not significantly, than among females. Significant familial clustering (genealogical index of familiality, $P=0.001$ ) was observed among the affected animals. Further subgroups were identified with significant $(P<0.001)$ aggregation among close relatives using kinship-based methods. Our analysis, along with the slightly higher disease frequency in males, suggests that dysautonomia may have a genetic causal factor with an $\mathrm{X}$-linked recessive inheritance pattern. This is the first study providing ancestry data and suggesting a heritable component in the likely multifactorial aetiology of the disease.
\end{abstract}

\section{KEYWORDS}

grass sickness, dysautonomia, heritable, equine, horse

\section{INTRODUCTION}

Equine dysautonomia (also known as equine grass sickness, EGS) is a life-threatening disease of unknown aetiology, affecting horses. Approximately $80 \%$ of the affected animals die within days after the recognition of the first clinical signs; the mortality is reported as $100 \%$ in the acute and subacute forms and 50\% in the chronic form (McGorum and Pirie, 2018). This polyneuropathy affects both the central and the peripheral nervous system and leads to severe clinical signs, due to gross and microscopic pathological alterations in the ganglions and neurocytes localised primarily in the gastrointestinal tract. Since its first recognition in 1909 
(Tocher et al., 1923), multiple cases have been reported around the world (McGorum and Pirie, 2018; Piccinelli et al., 2019).

Despite significant effort and research, neither the causative reason nor the pathomechanism of the disease is well understood. The most widely accepted hypothesis is a possible toxicoinfection by Clostridium botulinum type C, which was detected in the gut microflora of dissected horses (Hunter et al., 1999). However, studies performed to vaccinate horses against the bacteria have yielded mixed results, as both successful (McCarthy et al., 2004) and unsuccessful (Nunn et al., 2007a) vaccination attempts have been reported. Decades of research have associated several factors with an increased or decreased risk of the disease: phenotypic (age, body condition), environmental (soil nitrogen content, grass, number of horses, presence of birds, ruminants, younger animals, etc.), management (grazing, movement, stress, anthelmintic use, supplementary feeding, etc.) and climatic factors (cool, dry weather, frosts, increased number of hours of sunshine, maximum temperature, snowing) have been described (Doxey et al., 1991a, 1991b; Wood et al., 1998; McCarthy et al., 2004; Newton et al., 2004; Edwards et al., 2010; Wylie et al., 2014, 2016; McGorum and Pirie, 2018). The prevalence rate of the disease differs by country, but EGS can cause high morbidity $(10-25 \%)$ and high mortality $(50-100 \%)$ rates within a single herd, resulting in significant economic losses. Until recently, family patterns and pedigree analysis could not be performed as the majority of infected animals were of different origin.

The aims of the present study were (a) to perform a pedigree analysis on a study population affected by EGS, (b) to detect signs of heritability of EGS and (c) to show the possibility of genetic predisposition to EGS.

\section{MATERIALS AND METHODS}

\section{Study population}

The original goal of the study was to investigate a significant health issue of horses in a Hungarian horse stud, which has bred purebred Lipizzaner horses for more than 200 years. From 2001 onwards, 49 horses died due to EGS, resulting in significant economic and population-wide losses. Initial autopsy and pathological findings performed in 2001-2002 reported a diagnosis of the disease. Veterinary records showed that before their death, all of the affected animals were in a normal body condition, without advance indication of the severe colic signs typical of EGS. There have been several attempts to treat the affected animals, but the losses could not been prevented. A detailed description of the early observations has been published previously (Schwarz et al., 2012; Vörös et al., 2013). In the present study, the pedigree data of a total of 1,233 horses, born between 1996 and 2017, were analysed.

The Lipizzaner stud farm involved in this study is situated in the Bükk Mountains, North-eastern Hungary.
The assessed average population consisted of 70 broodmares, 12 stallions, approx. 150 weanlings and yearlings from the last breeding seasons and 40 non-breeding horses at the time of the evaluation (2016-2018). A total of approx. 280 horses are kept in three different areas close to each other in the stud. The affected groups are held in separate facilities based on their sex (central farm broodmares and weanlings and yearlings; colt farm - colts above 1 year of age; filly farm - fillies above 1 year of age). The animals affected with EGS (diagnosed as 'ill') were among the 1- to 4-year-old young horses of both sexes. The diagnosis of EGS was made after the veterinary records based upon pathological (dissection and histopathological) investigation. The studied population of horses was kept and fed under the same conditions (approx. $2 \mathrm{~kg}$ oats and $6 \mathrm{~kg}$ grass hay daily, with unlimited access to water). The mares and younger horses (aged $<4$ years) were moved to a pasture (located approx. $3 \mathrm{~km}$ away) twice daily. During the studied period (from 1996 until 2017) the stud was a closed herd, and no individuals were introduced to the population.

After the initial outbreaks of the disease in 2001, multiple attempts have been made to investigate the possible reason for the significant losses. Between 2001 and 2017, the water, the feed, the grass and grass hay, the soil, etc. were examined for toxins/dangerous materials but all investigations provided 'negative' results.

\section{Data collection}

Pedigree data for the analysed population (1996-2017) was provided by the Hungarian Association of Lipizzaner Horse Breeders and the Directorate for Animal Breeding (by Barnabás Szabó, National Food Chain Safety Office). Health and pathology data (veterinary records between 1996 and 2017) of 1,233 individuals were collected from the stud archives in order to find all EGS cases that had occurred during the study period.

\section{Vaccination trial}

Before the analysis of study data, a vaccination trial was performed on the 1- to 3-year-old foals in order to try to avoid further losses. This trial was not part of the present study, but the protocol and the results are described here to give more information for the reader.

Fifty fillies and colts from the affected stud were immunised with a 3-element vaccination programme against C. botulinum Type $\mathrm{C}$ toxoid (Botulismus Vakzine ad us. vet., Provet AG, Switzerland) and were regularly controlled by blood sampling and EGS ELISA measurements (Animal Health Trust Contract Research Group Laboratory, Newmarket, UK) as follows. The first vaccine was applied in June 2015 after the first vaccination treatment (T0). Thirty days (T30), 44 days (T44) and 210 days (T210) after the first immunisation, blood samples were taken and assayed for IgG levels in sera. At T30 and T44, a second and a third booster vaccine injection, respectively, was administered to the horses. 


\section{Statistical analyses}

For testing the sex independence of the EGS condition, Fisher's exact test was used (Agresti, 2007). The confidence interval of prevalence was estimated by Wilson's method (Agresti and Coull, 1998). The family aggregation was studied by genealogical index analysis (Hill, 1980), kinship sum test, kinship group ratio test (Rainer et al., 2016; Dinya and Solymosi, 2016) and familial incidence rate (FIR) test (Kerber, 1995). The method of Wellmann et al. (2012) was applied to estimate the time at risk required by the FIR test, since the amount of time spent in the stud was unknown for numerous animals. In FIR analysis, to handle the multiple testing issue the method of Benjamini and Hochberg (1995) was applied. While the genealogical index analysis is reported for all the samples, the kinship sum test, kinship group ration test and FIR test are calculated for each individual. All statistical analyses and visualisations were performed in R (R Core Team, 2018) using the packages FamAgg (Rainer et al., 2016), kinship2 (Sinnwell and Therneau, 2020) and optiSel (Wellmann, 2020).

\section{RESULTS}

Among the 1,233 animals in the study population, 49 were affected with EGS. The number of registered cases ranged between 1 and 11 yearly since 2001 (Fig. 1). The mean age of the diseased animals was 2.45 years (SD: 0.54 ). The overall prevalence of the disease was 3.97\% (95\% CI: 2.99-5.22). The average percentage of pedigree completeness was 0.79 (SD: 0.36), the median of the maximum pedigree depth was 6 (IQR: 5), the mean inbreeding coefficient was 0.0086 (SD: $0.016)$. Nonetheless, EGS occurred with significantly higher frequency among the progeny of certain males. For instance, as shown in Fig. 2, among the 32 descendants of stallion no. 719 , there were 7 affected horses $(21.9 \%$, 95\% CI: $10.46-$ 38.95). By performing familial aggregation test based on the kinship sums, and selecting individuals using the adjusted $P$ values because of multiple testing, we found that mare no. 933 as a member of pedigree, is highly connected to other affected members. Checking the ascendants of this mare, her parents, grandparents and great-grandparents had consanguineous matings. With the kinship group ratio test, we found three subsets in the pedigree where the observed

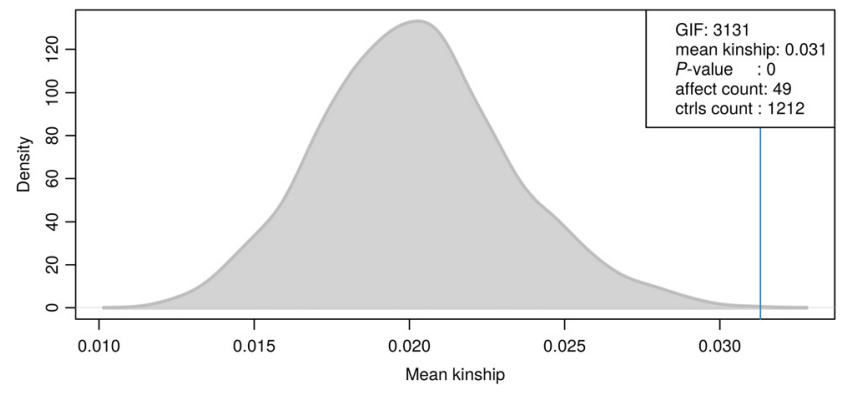

Fig. 2. Results of genealogical index analysis. The mean kinship, presented by the blue line, is the average of the kinships calculated for affected animals. The genealogical index of familiality (GIF) is the mean kinship multiplied by 100,000. For testing, the observed GIF simulations were performed by sampling the control animals. From these simulations, the expected mean kinship distribution is shown by the grey density plot. Since the blue line is far from the centre of the grey area, the probability of their common distribution is very low $(P=0.001)$

number of the affected individuals was significantly higher (adj. $P<0.001$ ) than expected. By FIR test, we found 82 individuals having significant (adj. $P<0.001$ ) FIR values, the highest FIR value (0.019) belonged to animal no. 719.

Comparing the rate of disease occurrence in males (28/ 539) and females (21/694), we found that the males have higher odds to be affected (OR: 1.76, 95\% CI: 0.95-3.29, $P=$ 0.057 ) although the difference was not significant. No outstanding frequencies were found when analysing the offspring of mares. With the genealogical index analysis, we found that the mean kinship is significantly higher [genealogical index of familiality (GIF): 3130.71 , mean kinship: 0.031 , $P=0.001]$ among the affected animals than its expected value (Fig. 3). Figure 4 shows the different proportions of the affected offspring of stallions having any diseased descendant.

Unfortunately the vaccination trial brought limited success, because the majority of immunised horses showed weak and variable immune responses (below the positive EGS ELISA reference optical density value). Furthermore, two colts from the vaccination group died of EGS in 2017.

\section{DISCUSSION}

Nowadays, EGS occurs worldwide except for Africa and Asia. In the years between 2000 and 2009, 141 cases were

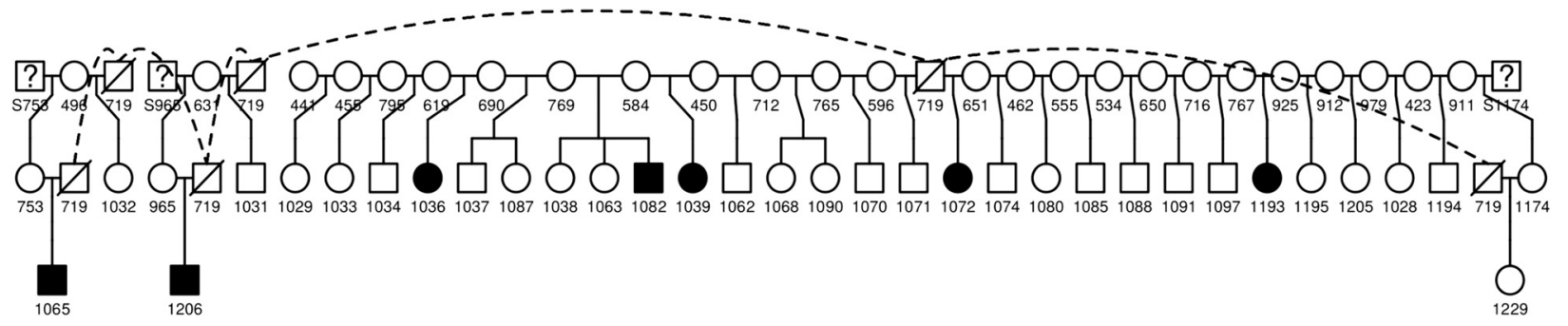

Fig. 1. The family tree of stallion no. 719 (crossed box) - the black nodes represent the affected, the white ones indicate the unaffected offspring. The circles and the rectangles depict the mares and stallions, respectively. The dashed lines highlight that the connected nodes represent the same animal (no. 719) in the pedigree, demonstrating the inbreeding 


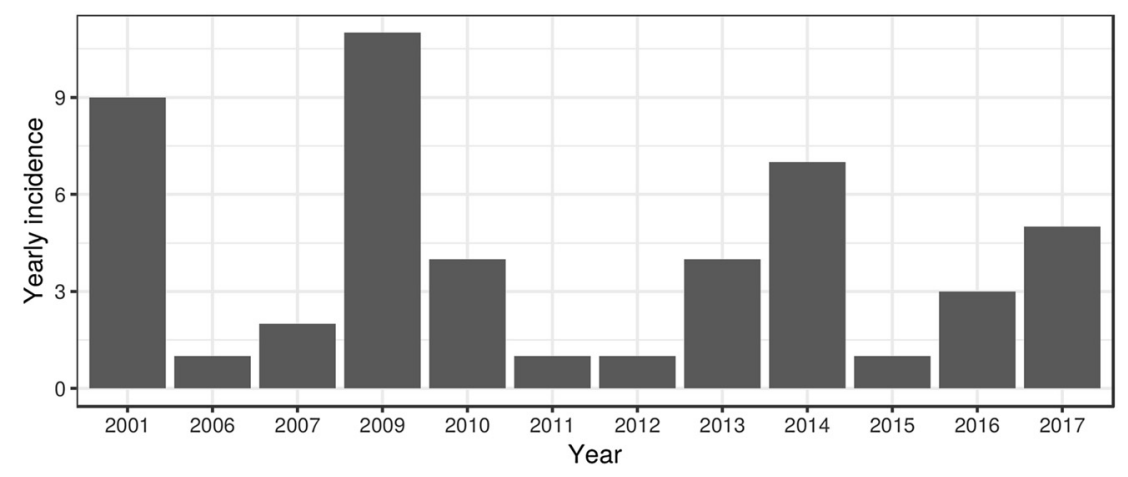

Fig. 3. The yearly incidence of grass sickness in the study population

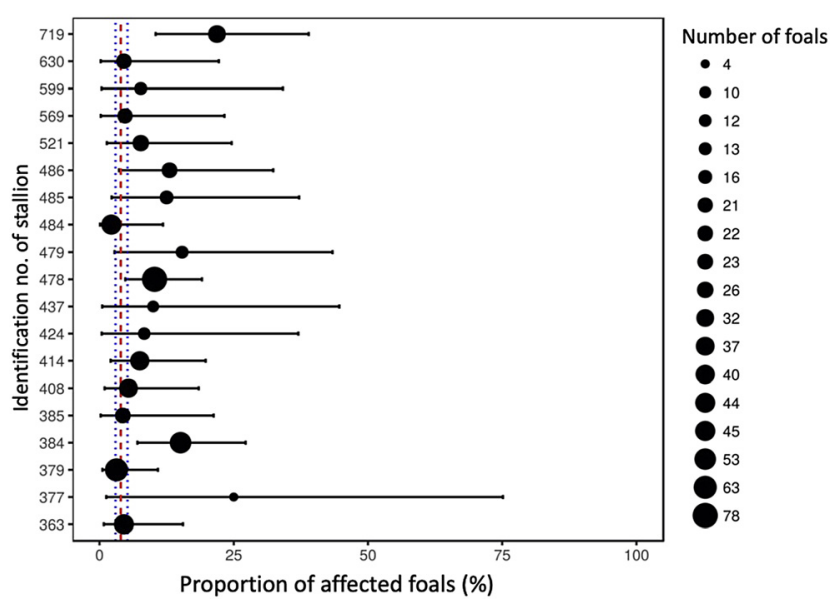

Fig. 4. The proportion of the affected offspring of stallions having any diseased descendant. The size of the dots represents the number of successors of a certain stallion. The horizontal lines show the $95 \%$ confidence interval of the affected proportions. The red vertical, dashed line indicates the overall prevalence of the disease in the population, while the blue dotted lines are its 95\% confidence interval

reported annually on average in Great Britain (Wylie et al., 2011). According to McCarthy et al. (2001), the disease can occur in three clinical forms but with different frequencies (47\% acute, $20 \%$ subacute and $33 \%$ chronic).

The diagnosis of EGS is based on the clinical and histopathological findings as described elsewhere (Milne, 1991; Cottrell et al., 1999; Pirie et al., 2014).

In the past century, many researchers attempted to explore the aetiology and pathophysiology of the disease. Various predisposing factors have been analysed (age, breed, stress, change in environmental conditions, season, weather, etc.) and numerous possible causative agents have been investigated (viruses, bacteria and their toxins, mycotoxins, toxic plants, chemicals, nutritional factors, insects, bugs, antiparasitic drugs, etc.) by researchers (McCarthy et al., 2001). None of these was found to successfully reproduce the disease.

The most common theory among researchers regarding the cause of the disease is the 'botulism theory'. Many studies concluded that C. botulinum, which produces toxins in the intestines of horses, can cause the disease, and some investigations showed higher prevalence of this bacterium in the samples of affected horses (Murray et al., 1994; Wood et al., 1998, 1999; Nunn et al., 2007a, 2007b). Moreover, the lesions caused by this toxin (BoNT-C) in the nerve endings of the affected horses were similar to those described in rat tissue studies in vitro (Williamson et al., 1995). Systemic antibody levels after vaccination against C. botulinum showed favourable results in some case-control studies (Hunter et al., 1999; Hunter and Poxton, 2001). Between 2013 and 2016, a vaccination trial was performed on the study population as well. However, after a 3-element (two basic immunisation injections and a booster) vaccination protocol and checking of the immunoglobulin levels, vaccinated horses showed high variability in their immune response and blood IgG levels. Therefore, the trial was interpreted as 'unsuccessful' by the stud director and the referring microbiology expert. Unfortunately two vaccinated foals died of EGS at 3 years of age. Therefore, the vaccination did not prevent the occurrence of EGS in this stud (personal communication - Dr. Orsolya Kutasi).

The disease is most prevalent in horses between 2 and 7 years of age, with a peak at the age of 4-5 years (McCarthy et al., 2001). In the present study, the mean age of the diseased animals was $2.45 \pm 0.54$ years which agrees with other reported findings. Although there was no sex predisposition in the previous studies (Wylie et al., 2014), we found higher odds in males than in females. This might indicate that a possible $\mathrm{X}$-linkage can be considered for the proposed inheritable disease disposition; however, the X-linked association could not be confirmed because of the high number of consanguineous mating events in the pedigree.

The overall disease prevalence in our study population is in good agreement with those found in previous studies in Great Britain (Cottrell et al., 1999), but among the descendants of some stallions (e.g. no. 719) the number of diseased foals (7/32) resulted in a frequency $(21.9 \%)$ much higher than the population average.

Testing the familial aggregation is the first step in the identification of possible genetic determinants of a disease (Matthews et al., 2008). If aggregation is found, more refined genetic studies are needed to identify specific chromosomal characteristics (e.g. mutations) that can be linked to the 
disease. In this study, one sick mare (no. 933) was found to be highly connected to other sick animals based on kinship analysis. By evaluating the pedigree of mare no. 933 we found numerous consanguineous mating events leading to inbreeding. The FIR test showed a significant (adj. $P<0.05$ ) FIR value among affected animals; therefore, we may conclude that inbreeding causes the aggregation (phenotypic appearance) of EGS.

The present study has the limitation that it examines the population data of only a single Hungarian stud; this should be taken into consideration when extrapolating the results.

To summarise, it is always a hard task to determine whether or not a disease is heritable. A thorough pedigree analysis should be performed as the first step to examine a possible aggregation in the family. The methods of statistical analysis differ, and none of them can verify a genetic background individually; therefore, we used several methods during the investigation. Based on the results presented in this paper, the authors think that the situation is likely complicated - the predisposition might be inherited and may be an additional factor needed for the clinical signs to occur. In the literature of EGS and in the study population, stress (e.g. a change in environment) as a factor is likely to be present before the clinical signs develop. Recent transportation, feed change, cold, dry weather, irregular frosts (McGorum and Pirie, 2018) and storms are mentioned as possible factors predisposing horses to EGS.

To improve our understanding of the genetic background of the disease, a genome-wide association study (GWAS) should be performed to find differentiating genes and polymorphisms (Bush and Moore, 2012). If such causative features are found, further works may lead to a practically applicable genetic test. If such a test were available, the genetic predisposition would be identifiable in horses, and a breeding selection based on the test results could be made to avoid the phenotypic appearance of EGS in the near future.

\section{ACKNOWLEDGEMENTS}

The authors would like to thank Barnabás Szabó, Péter Marlok, István Péntek (National Food Chain Safety Office), Dr. Balázs Pataki and Dr. András Gáspárdy for their help in the initial pedigree data collection and Andor Dallos $\nmid$ for his continuous help in collecting health data records covering 16 years in the stud. The study was supported through the New National Excellence Program of the Ministry of Human Capacities of Hungary (Ms Márta Varga).

\section{REFERENCES}

Agresti, A. (2007): Categorical Data Analysis. 2nd ed. John Wiley \& Sons, New York.

Agresti, A. and Coull, B. A. (1998): Approximate is better than 'exact' for interval estimation of binomial proportions. Am. Statistician 52, 119-126.
Benjamini, Y. and Hochberg, Y. (1995): Controlling the false discovery rate: a practical and powerful approach to multiple testing. J. Roy. Stat. Soc. B 57, 289-300.

Bush, W. S. and Moore, J. H. (2012): Chapter 11: genome-wide association studies. PLoS Comput. Biol. 8, 1-11.

Cottrell, D. F., McGorum, B. C. and Pearson, G. T. (1999): The neurology and enterology of equine grass sickness: a review of basic mechanisms. Neurogastr. Motil. 11, 79-92.

Dinya, E. and Solymosi, N. (2016): Biometry in Clinics 2. Solving Tasks in R-environment [in Hungarian]. Medicina Könyvkiadó Zrt., Budapest.

Doxey, D. L., Gilmour, J. S. and Milne, E. M. (1991a): A comparative study of normal equine populations and those with grass sickness (dysautonomia) in eastern Scotland. Equine Vet. J. 23, 365-369.

Doxey, D. L., Gilmour, J. S. and Milne, E. M. (1991b): The relationship between meteorological features and equine grass sickness (dysautonomia). Equine Vet. J. 23, 370-373.

Edwards, S. E., Martz, K. E., Rogge, A. and Heinrich, M. (2010): Edaphic and phytochemical factors as predictors of equine grass sickness cases in the UK. Front. Pharmacol. 1, 122.

Hill, J. R. (1980): Cancer incidence in defined populations, volume 4 of Banbury report, chapter A: survey of cancer sites by kinship in the Utah Mormon population. Cold Spring Harbor Laboratory, Cold Spring Harbor, New York. pp. 299-318.

Hunter, L. C. and Poxton, I. R. (2001): Systemic antibodies to Clostridium botulinum type C: do they protect horses from grass sickness (dysautonomia)? Equine Vet. J. 33, 547-553.

Hunter, L. C., Miller, J. K. and Poxton, I. R. (1999): The association of Clostridium botulinum type $\mathrm{C}$ with equine grass sickness: a toxicoinfection? Equine Vet. J. 31, 492-499.

Kerber, R. A. (1995): Method for calculating risk associated with family history of a disease. Genet. Epidemiol. 12, 291-301.

Matthews, A. G., Finkelstein, D. M. and Betensky, R. A. (2008): Analysis of familial aggregation studies with complex ascertainment schemes. Stat. Med. 27, 5076-5092.

McCarthy, H. E., French, N. P, Edwards, G. B., Poxton, I. R., Kelly, D. F., Payne-Johnson, C. E., Miller, K. and Proudman, C. J. (2004): Equine grass sickness is associated with low antibody levels to Clostridium botulinum: a matched case-control study. Equine Vet. J. 36, 123-129.

McCarthy, H. E., Proudman, C. J. and French, N. P. (2001): Epidemiology of equine grass sickness: a literature review (1909-1999). Vet. Rec. 149, 293-300.

McGorum, B. C. and Pirie, R. S. (2018): Equine dysautonomia. Vet. Clin. N. Am. Equine Pract. 34, 113-125.

Milne, E. (1991): Grass sickness. Equine Vet. Educ. 3, 196-199.

Murray, A, Cottrell, D. F. and Woodman, M. P. (1994): Cholinergic activity of intestinal muscle in vitro taken from horses with and without equine grass sickness. Vet. Res. Commun. 18, 199-207.

Newton, J. R., Hedderson, E. J., Adams, V. J., McGorum, B. C., Proudman, C. J. and Wood, J. L. N. (2004): An epidemiological study of risk factors associated with the recurrence of equine grass sickness (dysautonomia) on previously affected premises. Equine Vet. J. 36, 105-112.

Nunn, F. G., Pirie, R. S., McGorum, B. C., Wernery, U. and Poxton, I. R. (2007a): Comparison of IgG antibody levels to Clostridium botulinum antigens between euthanased and surviving cases of chronic grass sickness. Res. Vet. Sci. 83, 82-84. 
Nunn, F. G., Pirie, R. S., McGorum, B. C., Wernery, U. and Poxton, I. R. (2007b): Preliminary study of mucosal IgA in the equine small intestine: specific IgA in cases of acute grass sickness and controls. Equine Vet. J. 39, 457-460.

Piccinelli, C., Jago, R. and Milne, E. (2019): Ganglion cytology: a novel rapid method for the diagnosis of equine dysautonomia. Vet. Pathol. 56, 244-247.

Pirie, R. S., Jago, R. C. and Hudson, N. P. H. (2014): Equine grass sickness. Equine Vet. J. 46, 545-553.

R Core Team (2018): R: A Language and Environment for Statistical Computing. R Foundation for Statistical Computing, Vienna, Austria. URL https://www.R-project.org/.

Rainer, J., Taliun, D., D'Elia, Y., Pattaro, C., Domingues, F. S. and Weichenberger, C. X. (2016): FamAgg: an R package to evaluate familial aggregation of traits in large pedigrees, Bioinformatics 32, 1583-1585.

Schwarz, B., Brunthaler, R., Hahn, C. and van den Hoven, R. (2012): Outbreaks of equine grass sickness in Hungary. Vet. Rec. 170, 75.

Sinnwell, J. and Therneau, T. (2020): Kinship2: pedigree functions. $\mathrm{R}$ package version 1.8.5. URL https://CRAN.R-project.org/ package $=$ kinship 2 .

Tocher, J. F., Brown, W., Tocher, J. W. and Buxton, J. B. (1923): Grass sickness investigation report. Vet. Rec. 3, 75-89.

Vörös, K., Bakos, Z., Albert, M., Barátossy, Gy. and Fejér, B. (2003): Occurrence of grass sickness in Hungary [in Hungarian, with English abstract]. Magy. Allatorvosok 125, 67-74.
Wellmann, R. (2020): optiSel: optimum contribution selection and population genetics. $\mathrm{R}$ package version 2.0.3. URL https:// CRAN.R-project.org/package $=$ optiSel.

Wellmann, R., Hartwig, S. and Bennewitz, J. (2012) Optimum contribution selection for conserved populations with historic migration. Genet. Sel. Evol. 44, 34.

Williamson, L. C., Halpern, J., Dunlap, V. and Neale, E. A. (1995): Botulinum neurotoxin $\mathrm{C}$ acts on syntaxin and SNAP-25 and is cytotoxic to neurons. Mol. Biol. Cell 6, 61.

Wood, J. L., McGorum, B. C. and Mayhew, I. G. (1999): Equine dysautonomia: has grass been blamed unfairly all this time? Equine Vet. J. 31, 451-452.

Wood, J. L., Milne, E. M. and Doxey, D. L. (1998): A case-control study of grass sickness (equine dysautonomia) in the United Kingdom. Vet. J. 156, 7-14.

Wylie, C. E., Proudman, C. J., McGorum, B. C. and Newton, J. R. (2011): A nationwide surveillance scheme for equine grass sickness in Great Britain: results for the period 2000-2009. Equine Vet. J. 43, 571-579.

Wylie, C. E., Shaw, D. J., Fordyce, F. M., Lilly, A. and McGorum, B. C. (2014): Equine grass sickness in Scotland: a case-control study of signalment- and meteorology-related risk factors. Equine Vet. J. 46, 64-71.

Wylie, C. E., Shaw, D. J., Fordyce, F. M., Lilly, A., Pirie, R. S. and McGorum, B. C. (2016): Equine grass sickness in Scotland: a case-control study of environmental geochemical risk factors. Equine Vet. J. 48, 779-785. 